\title{
Numerical analysis of the electrical conduction in carbon nanostructures
}

\author{
M. Britch, K. Dobrego, L. Krasovskaya \\ Heat and Mass Transfer Institute, NASB, Belarus
}

The problem of the single molecule conductance measurement and calculation attracts much attention due to its possible use in the molecular electronics. Another promising line of research is connected with consideration of carbon nanostructures, in particular graphene and nanotubes, as possible elements of nanoelectronic devices. Here, we present the results of modeling of the conduction in systems including fullerenes. Among other members of the carbon nanostructures family, the fullerenes have advantages of relatively low cost and suitability for mass production and manipulation. The size of the fullerenes and their spacing in the fullerite crystal are appropriate to form quantum dots possessing specific physical properties that may be useful for many applications. The systems consisting of the single fullerene molecule or a simplecubic lattice of the fullerite placed between two metal electrodes has been considered. The electric current as a function of the bias voltage was calculated using the Landauer formula, connecting the charge transport with the transmission of electrons. To obtain the transmission matrix components the supplementary problem of the electron scattering by the model potential had been solved. The transmission probability as a function of the electron energy and the electric current as a function of the bias voltage have been calculated. Our simulation has shown that the current - voltage characteristics of systems including fullerenes have negativeconductance regions that makes it possible to use them as active elements of nanoelectronic devices (particularly, generators of the RF-radiation).

NOTES

\section{Non-plasmonic hyperthermia: prerequisites for realization and materials}

\author{
Postnikov ${ }^{1}$, K. Moldosanov ${ }^{2}$ \\ ${ }^{1}$ Université de Lorraine, Institut de Chimie, Physique et Matériaux, France; \\ ${ }^{2}$ Kyrgyz-Russian Slavic University, Kyrgyzstan
}

Nanoparticles of biologically compatible alloys with intensive electron-electron scattering are considered as potential substitutes for gold nanoparticles and silica-gold nanoshells in the photothermal cancer therapy. In this approach, it is proposed to heat the nanoparticles at the expense of the Joule heat generation, rather than due to plasmon excitation. Correspondingly, it is suggested that the resonance absorption of the laser radiation should be achieved due to specificity of the electron structure of the nanoparticle material, namely due to an enhanced electron density of states at the Fermi energy and also within the energy range 1.3 to 1.9 eV, i.e., in the transparency window of the biological tissues, above it. Estimations show that application of nanoparticles of the alloys with intensive electron-electron scattering can improve an efficiency of the laser radiation energy-to-heat conversion. In this case, the nanoparticles need not to have neither special cores nor the rice grain-like shape. The manufacture of the uniform spherical coreless nanoparticles is simpler and lower in cost. It seems likely that in the non-plasmonic hyperthermia, besides the gold alloys, the other biologically compatible metals and alloys can be applied (for example, tantalum and its alloys, and also the NiTi alloy).

NOTES 